\title{
Zur 2. Auflage
}

Die Initiative des Verlages ist es gewesen, welche die zweite Auflage der "Geschichte der altchristlichen Literatur" Adolf Harnacks zuwege gebracht hat. Wir leben in einem Zeitalter der Neudrucke wissenschaftlicher Standardwerke. In allen geisteswissenschaftlichen Disziplinen erscheinen sie in ständig wachsender Zahl: um die Lücken auszufüllen, welche der zweite Weltkrieg in die Bestände der Bibliotheken gerissen hat, um dem Bedarf der allerorts neu entstehenden wissenschaftlichen Institutionen $\mathbf{z u}$ genügen - und um unser Unvermögen zu überbrücken, Neues zu schaffen, welches das Alte gleichwertig ersetzen könnte. Alle diese Voraussetzungen treffen auf Harnacks Werk zu. So geht es in neuem Gewande in die Welt hinaus; der Aufforderung, ihm ein Geleitwort mitzugeben, konnte sich der Unterzeichnete trotz verständlichen Zögerns nicht entziehen, steht es doch in zu enger Verbindung mit den von ihm seit längeren Jahren geleiteten Arbeiten der heutigen Kommission für spätantike Religionsgeschichte an der Deutschen Akademie der Wissenschaften zu Berlin.

Uber den äußeren Anlaß zur Entstehung seiner „Geschichte der altchristlichen Literatur" berichtet Harnack selbst in der Vorrede zur ersten Auflage (S. V), daß der Vorschlag dafür von ihm im Zusammenhang der Vorbereitungen für die Ausgabe der „Griechischen Christlichen Schriftsteller" gemacht worden sei. Am 22. Januar 1891, so erfahren wir aus den glücklicherweise erhaltenen Akten der Akademie, legte Harnack der phil.hist. Klasse den Entwurf eines entsprechenden Antrages vor, dem die Klasse einmütig zustimmte:

„Ew. Excellenz hat die K. Akademie der Wissenschaften in ihrem Bericht vom 21. Juni [1890] ehrerbietigst davon in Kenntniß gesetzt, daß sie die Absicht hegt, ihren wissenschaftlichen Unternehmungen ein Corpus Patrum Graecorum Antenicaenorum, d. h. eine Ausgabe der gesammten christlichen vornicänischen Litteratur in griechischer Sprache, hinzuzufügen. Auf die Nothwendigkeit und Wichtigkeit eines solchen Unternehmens glaubt die Akademie nicht besonders hinweisen zu müssen. Die Wiener Akademie der Wissenschaften arbeitet seit mehr als 25 Jahren an einer Gesammtausgabe der lateinischen Kirchenväter. Die Aufgabe, welche die K. Akademie sich stellt, ist dem gegenüber eine viel begrenztere, aber in Hinsicht auf die geschichtliche Bedeutung der betreffenden Litteratur eine noch wichtigere - es sollen alle litterarischen Denkmäler des ältesten 
Christentums von seiner Entstehung bis zur Begründung der Reichskirche durch Konstantin (abgesehen von dem Neuen Testamente und den lateinischen Quellenschriften) herausgegeben werden.

Um dieses Unternehmen in der sichersten Weise zu begründen und zugleich die Kosten der späteren handschriftlichen Forschungen auf das nothwendigste $\mathrm{MaB}$ zu beschränken, erscheint es der K. Akademie der Wissenschaften zweckmäßig, zunächst eine genaue und detaillierte Ubersicht über das gesammte Material zu gewinnen, über das, was bereits auf diesem Gebiete geleistet ist und was noch zu geschehen hat. Eine solche Utbersicht kann bei dem gegenwärtigen Stande der Sache für die christliche Litteratur der ersten 3 bis 4 Jahrhunderte wesentlich, wenn auch nicht durchweg, befriedigend ohne Reisen auf Grund der gedruckten Handschriftencataloge und Bücher gewonnen werden. Eìn solcher Conspectus, der die gesammte vornicänische Litteratur genau verzeichnet und bei jedem Stück die Art der Erhaltung, die Handschriften und - in Kürze - die Geschichte der Uberlieferung angiebt, wird es ermöglichen, einen ausführlichen und sicheren Arbeitsplan für die Herausgabe der Schriften aufzustellen, Verzettelungen der Arbeitskräfte und der Mittel zu vermeiden und mit den geringsten Opfern an Zeit und Geld das Beste zu leisten. Es würde auch an sich eine sehr nützliche Leistung auf dem Gebiete der Litteraturgeschichte sein, selbst wenn die geplante Ausgabe nicht zu Stande kommen sollte. Eine solche Ubersicht - Prolegomena zur altchristlichen Litteraturgeschichte und zu einer Gesammtausgabe - läßt sich unseres Erachtens in c. zwei Jahren herstellen."

Bereits am 12. Februar 1891 wurde die offizielle Zustimmung gegeben, am Tage darauf begann die damalige Kirchenväterkommission der PreuBischen Akademie der Wissenschaften ihre Arbeit. Charakteristisch genug, daß man sich nicht mit formellen Sitzungen die Zeit wegnahm - die erste eigentliche Sitzung der Kommission fand erst 6 Jahre später, am 20. März 1897, statt - Harnack unterrichtete die Mitglieder vielmehr lediglich durch Rundschreiben über den jeweiligen Stand der Dinge, zu denen sie in Randnotizen ihre Ansichten äußerten. Vielmehr wurde alle Kraft auf die eigentliche Arbeit konzentriert. Auf den Tag genau hielt Harnack seine Zusage ein: am 15. Januar 1893 befand sich der Anfang des Manuskriptes schon in der Druckerei und war das Manuskript für die gesamte „Uberlieferung" (d. h. die beiden ersten Bände des ganzen Werkes) so gut wie abgeschlossen. Noch im selben Jahre kamen diese Bände heraus. Die beiden Bände der „Chronologie“ folgten vier bzw. elf Jahre später.

Auch wenn man berücksichtigt, daß Harnack schon lange vor 1891 mit der Sammlung des Materials - zunächst unter dem Gesichtspunkt anderer Arbeiten - begonnen hatte, bleibt es eine für unsere heutigen Maßstäbe schier unfaßbare Leistung, die er in den zwei Jahren von 1891 bis 1893 mit den 1000 Druckseiten der ,Utberlieferung " vollbracht hat. Zwar konnte ihm die Akademie in Erwin Preuschen für diese Zeit einen haupt- 
amtlichen Mitarbeiter stellen, zwar wußte er für Spezialabschnitte manchen anderen Helfer zu gewinnen (vgl. S. VII), aber zwei Drittel des gesamten Werkes stammen, wie wir aus der Korrespondenz jener Jahre wissen, ausschließlich von Harnack selbst, und auch am restlichen Drittel hat er oft genug selbst Hand angelegt.

Damals, als Harnacks "Geschichte der altchristlichen Literatur" zu erscheinen begann, stand sie allein auf dem weiten Feld. Heute, nach über 50 Jahren, besitzen wir die „Geschichte der altkirchlichen Literatur“ von 0. Bardenhewer, die Darstellung der ,altchristlichen griechischen Litteratur" von 0. Stählin in W. v. Christs "Geschichte der griechischen Litteratur: $"$, die parallele Behandlung der lateinischen christlichen $\mathrm{Au}$ toren in der "Geschichte der römischen Litteratur" von Schanz-Hosius durch G. Krüger (als Seitenstücke dazu die Abschnitte von E. Klostermann in W.S. Teuffels ,Geschichte der römischen Literatur"“, die Patrologien von Altaner, Steidle, Quasten u.a.m., wozu dann die Werke von A. Puech, Labriolle, Bardy und anderen kommen. Ist dadurch Harnacks Werk überholt und beiseitegeschoben oder auch nur in seiner Stellung gefährdet? Ich glaube nicht. Vielmehr stehen wir m. E. noch immer an der Stelle, die Harnacks Vorwort selbst bezeichnet. Man muß es nur in der Umschreibung der Aufgabe genau lesen. Harnack sagt dort (S. V), sein Ziel sei eine „Bibliotheca Antenicaena“ nach dem Vorbild der „Bibliotheca Graeca" von Fabricius gewesen:

„Den Zweck und Inhalt des vorstehenden Werkes bitte ich nach diesen Absichten zu beurtheilen. Es will das Material möglichst vollständig in allen Verzweigungen seiner Uberlieferung vorführen, es genau kenntlich machen und soweit gesichtet geben, als es die bisherige Forschung bereits gesichtet hat. Es will „Mémoires pour servir à l'histoire ecclésiastique“ darstellen und jedem küntigen Herausgeber irgend eines vornicänischen Denkmals ebenso nützlich sein wie dem Historiker und dem Freunde der ältesten Geschichte der Kirche. Es will endlich zukünftige Nachforschungen in den Handschriftenschätzen der Bibliotheken erleichtern und als zuverlässiges Hülfsmittel zur Identificirung dienen" (S. V/VI).

Nach dieser Zielsetzung und innerhalb dieser Grenzen hat Harnack gearbeitet. Keine der oben genannten Literaturgeschichten hat eine ähnliche Abzweckung. Am nächsten kommen Harnacks Arbeit noch das Werk von Bardenhewer und die Krügerschen Abschnitte über die lateinischen Väter bei Schanz-Hosius. In bezug auf Bardenhewer genügt es wohl Lietzmann zu zitieren, der Harnacks Arbeit durchaus kritisch gegenüber gestanden hat (vgl. seine Besprechung in der Theol. Rundschau 8, 1905, $345 \mathrm{ff}$.): „Man lernt also bei Harnack mehr, und das liegt doch nicht bloß daran, daß er mehr Platz hat" (ebda S. 349). Und im Schanzschen Handbuch werden lediglich die mit der Wende zum dritten Jahrhundert einsetzenden lateinischen christlichen Autoren behandelt, während der weit überwiegende Teil bei Harnack nach Lage der Dinge eben den griechischen 
Schriften und Schriftstellern gewidmet ist. 0 . Stählin war bei seiner mustergültigen Arbeit auf 400 Druckseiten beschränkt, schon von daher ist ein Vergleich mit Harnack nicht möglich. Und die modernen, erfreulicherweise regelmäßig in neuen Auflagen erscheinenden Patrologien dienen im wesentlichen dem Lehrbetrieb; wenn der Fortgeschrittene sie benutzt, dann zur Unterrichtung über neu erschienene Ausgaben und Literatur oder zur Auffrischung seines Gedächtnisses. Es ist aufrichtig zu beklagen, daß der von B. Altaner 1946 vorgetragene Plan (in den Miscellanea Giovanni Mercati I: „Der Stand der patrologischen Wissenschaft und das Problem einer neuen altchristlichen Literaturgeschichte") sich bisher nicht hat verwirklichen lassen und - wenigsten; soweit B. Altaner selbst in Betracht kommt, dessen angegriffene Gesundheit seiner Arbeit bedauerliche Grenzen zieht, - in absehbarer Zeit auch wohl kaum verwirklicht werden wird. Altaner erklärte damals seine Absicht, ,eine neue kritische vier- oder fünfbändige Geschichte der altchristlichen Literatur zu schreiben, die alle Hauptergebnisse der neuesten Forschung verarbeitet und dem Fachmann das notwendige Rüstzeug furr seine Weiterarbeit in die Hand gibt" (S. 33). Hier wäre ein Werk entstanden, welches Harnacks „Geschichte der altchristlichen Literatur", wenigstens in seinen ersten beiden Bänden, bis zum gewissen Grade ersetzt hätte. Bis zu einem gewissen Grade: denn in Anbetracht des vorgesehenen Umfangs hätte das bei Harnack gebotene Material nur in sehr eingeschränktem Maße vorgelegt werden können, und die beiden Bände der „Chronologie“ hätten ohnehin ihre Stellung behalten. Aber trotzdem: wir hätten bei der Ausführung des Altanerschen Vorhabens ein Handbuch besessen, das unserer Zeit weithin die Hilfe geboten hätte, deren wir dringend bedürfen. So aber stehen die Dinge heute durchaus noch so, wie Dräseke (dessen Gefühle Harnacks Werk gegenüber in Anbetracht dessen kritischer Stellungnahme zu eigenen Arbeiten durchaus temperiert waren) es 1893 (Wochenschrift für klassische Philologie 1893, 1329-1334) formulierte: ,mit dem Uberholtwerden wird es doch wohl vorläufig noch ziemlich lange gute Wege haben" (ebda Sp. 1331).

Das Wort, auf das Dräseke sich bezieht, steht am Ende der Vorrede Harnacks zur 1. Auflage des ersten Bandes:

„Eine Arbeit, wie die unsrige, steht leider unter dem Geschick, nie vollendet und bald überholt zu werden - sollte der Wahlspruch gelten: „Tout bien ou rien", so wäre sie nie geschrieben worden" (S. XIl).

Der zweite Teil dieses Wortes gilt in verstärktem Maße auch für die vorliegende zweite Auflage. Selbstverständlich sind, ehe die vorliegende Form gewählt wurde - sie stellt die äußerste Konzession des Verlages dar - Uberlegungen angestellt worden, ob nicht andere Wege möglich wären. Aber immer wieder bestätigte sich die von vornherein feststehende Erkenntnis, daß es völlig unmöglich sei, Harnacks Werk auf den gegenwärtigen Stand unserer Kenntnisse zu bringen. Das hätte bedeutet, es neu zu schreiben. Nicht nur der Unterzeichnete fühlte sich dazu nicht in der 
Lage (was keines Kommentares bedarf, denn er ist sich der Mangelhaftigkeit seiner Voraussetzungen dafür voll bewußt), - auch sonst, meinte er allerdings, sei niemand dazu imstande. Jeder, der auf dem Felde der alten Kirchengeschichte arbeitet, wird auf seinem Spezialgebiet mehr und vielleicht auch anderes zu sagen wissen als Harnacks Literaturgeschichte; für das Ganze aber kann das m. E. niemand von sich behaupten. Noch heute gilt, was Loofs bemerkte, dem wohl niemand mangelnde Akribie nachsagen kann und der wie Harnack wahrlich über große Materialsammlungen verfügte: ,Niemand hat gegenwärtig, was hier geboten wird; niemand hat Kollektaneen, die dies Buch ihm ersetzen könnten" (Deutsche Literaturzeitung 1894, Sp.389). Nicht daß wir nicht daran arbeiteten, manches neu zu machen, was an Harnacks Werk unbedingt neu gemacht werden muß. Die Kommission für spätantike Religionsgeschichte ist z. B., wie bekannt, nicht nur bemüht, die vor dem zweiten Weltkriege erschienenen und heute meist vergriffenen Bände der „Griechischen Christlichen Schriftsteller" durch revidierte Neuausgaben $\mathrm{zu}$ ersetzen und früher offen gelassene Lücken $\mathrm{zu}$ schließen, sie hat ihren Arbeitsbereich auch weit über die einst von Harnack gezogene Grenze von 325 hinausgeschoben. Die Basis für das alles ist die vollständige Feststellung des nun tatsächlich in aller Welt vorhandenen Handschriftenmaterials. Glücklicherweise verfügen wir heute, wenn die jetzt im Druck befindliche zweite Auflage von M. Richards Répertoire des bibliothèques et des manuscrits grees vorliegen wird, über sehr viel bessere Voraussetzungen als frühere Zeiten, um der Handschriftenkataloge und auch der griechischen Handschriften selbst habhaft zu werden. Systematisch diese Handschriften zu erfassen, sind wir nach Kräften bemüht. Aber lange Jahre werden vergehen, bis wenigstens ein ungefährer Abschluß dieser Arbeiten erreicht sein wird. Und dann ist nur ein Teilgebiet von den vielen neu erschlossen, die Voraussetzung dafür sind, daß Harnacks Werk einmal neu geschrieben werden kann. Auch der immer wiederholten Klage, daß man heute nicht mehr in der Lage sei, auch nur die Titel der in immer steigender Zahl erscheinenden Monographien und Ausgaben einigermaßen vollständig zusammen zu bekommen, ja daß man nicht einmal alle für die ersten Jahrhunderte in Betracht kommenden Zeitschriften überblicken könne, um von ihrer Lektüre zu schweigen, sucht die Kommission für spätantike Religionsgeschichte abzuhelfen. Im Zusammenwirken mit Kollegen aus verschiedenen Ländern bereitet sie gegenwärtig das Erscheinen einer Internationalen patristischen Bibliographie vor. Aber auch das ist nur ein kleiner Schritt voran auf dem langen Wege bis zu einer Erneuerung der Harnackschen „Geschichte der altchristlichen Literatur“.

Bis dahin werden wir uns also mit dem Vorhandenen begnügen müssen, und wir werden gut daran tun, es für jede unserer Arbeiten zu benutzen. Das ist nun nicht so gemeint, um Mißverständnissen vorzubeugen, daß Harnacks Werk als alleinige Grundlage gebraucht werden könnte, aber für jedermann, der über die ersten drei Jahrhunderte unserer Zeit- 
rechnung arbeitet, wird es Ausgangspunkt, unumgängliche Voraussetzung sein müssen. $\mathrm{Da} B$ in der vorliegenden zweiten Auflage eine nicht geringe Mühe aufgewandt ist, um das Werk von offenkundigen Versehen und Irrtümern des Druckers und auch des Verfassers zu reinigen, werden dem Benutzer die insgesamt 55 Seiten Berichtigungen und Nachträge zeigen, welche in der zweiten Auflage im Vergleich zur ersten bald auf das Dreifache gestiegen sind. Dabei sollte in jedem Fall - soweit möglich auch mit seinen Worten - die Meinung Harnacks selbst, nicht die des Herausgebers, zum Ausdruck gebracht werden. Das wurde dadurch erleichtert, daß Harnack in seinen Selbstanzeigen in der Theologischen Literaturzeitung $(18,1893,542-543 ; 22,1897,76-78 ; 29,1904,355-356)$ wie vor allem in Bd 12,1 der Texte und Untersuchungen (,Zur Uberlieferungsgeschichte der altchristlichen Lit'eratur", 1904) bereits Nachträge und Berichtigungen zu seiner Arbeit geliefert hat. Weiteres Material boten die größeren Rezensionen - etwa 40 an der Zahl - sowie das Handexemplar G. Krügers, das uns durch die Freundlichkeit von Prof. Dr. Adam/Bethel zugänglich wurde. Schon mehrere der Kritiker Harnacks wurden zitiert (die zahlreichen Lobredner blieben mit Absicht ausgeschlossen). An den Schluß möge G. Krügers Urteil gestellt werden, der wie kein anderer seine von Harnack abweichenden Meinungen zum Ausdruck gebracht hat. Allein seine Besprechung von Harnacks Band II, 2 in den Göttinger Gelehrten Anzeigen umfaßt 56 Seiten! (GGA 167, 1905, 1-56). Aber selbst sie steht unter dem Vorzeichen: „daß solcher Widerspruch die Dankbarkeit des Schülers zur Folie hat, der auf diesem Gebiete fast alles von Harnack empfangen zu haben immer wieder dankbar bekennen muß" (ebda S. 3). $\mathrm{Da} ß$ aus diesen Kritiken nur Sachangaben übernommen wurden und nicht die jeweiligen persönlichen Auffassungen der Kritiker, versteht sich. Ebenso blieben Literaturnachträge - sofern sie nicht auf Harnack selbst zurückgingen - ausgeschlossen: wo anfangen und wo aufhören? Wo sich durch Änderungen in den Seitenzahlen die Notwendigkeit von Umstellungen in den Registern ergab, sind diese im fotomechanischen Nachdruck durch Tekturen vorgenommen worden. In einigen Fällen war das nicht möglich. Dann ist durch ein am Rande der Seite angebrachtes Sternchen angegeben, daß eine Fortsetzung im Registernachtrag zu suchen ist. Auf diese Weise werden dem Benutzer des Werkes auch die Nachträge am bequemsten erschlossen.

F. Winkelmann, Mitarbeiter der Kommission für spätantike Religionsgeschichte, bin ich für seine Hilfe bei dem mühseligen wie entsagungsvollen Unternehmen der Neuauflage zu Dank verpflichtet.

Halle-Berlin, den 1. September 1957 